Praxis, Vol. 14, No. 2, 253 -263

DOI: http://dx.doi.org/10.21676/23897856.2886

\title{
Sostenibilidad y educación en el área de tecnología e informática
}

\section{Sustainability and education in the area of technology and informatics}

\author{
Silvia Andrea Torres Oliveros ${ }^{1}$ (D) Julieth Salcedo Ospino² ${ }^{\text {D }}$, Lucía Bustamante Meza ${ }^{3}$ iD
}

Tipología: artículo de reflexión

Para citar este artículo: Torres, S., Salcedo, J. y Bustamante, L. (2018). Sostenibilidad y educación en el área de tecnología e informática. Praxis, 14(2), 253-263. Doi: http://dx.doi.org/10.21676/23897856.2886

Recibido en diciembre 1 de 2018

Aceptado en diciembre 16 de 2018

Publicado en línea en diciembre 16 de 2018

\section{RESUMEN}

El presente tiene como objetivo reflexionar sobre el nivel de conocimientos en sostenibilidad de los estudiantes de Licenciatura en Informática de la Universidad del Magdalena y su responsabilidad para promover el manejo adecuado de RAEE. Tal propósito surge del preocupante hecho de que los avances tecnológicos son valorados por los beneficios e innovación que han traído, restando importancia a las consecuencias que generan sus residuos, los cuales están compuestos por materiales tanto valiosos como potencialmente peligrosos. Entre los principales referentes teóricos considerados están los ODS de la UNESCO, los lineamientos técnicos para manejo adecuado de RAEE, el Libro verde de Colciencias, que promueven desarrollar competencias en sostenibilidad como eje transversal en los diferentes niveles educativos. Las reflexiones descritas surgen de una investigación descriptiva cuya población fueron estudiantes de la asignatura Fundamentos de Programación durante el 2018, a quienes se les aplicó una prueba piloto en la que se determinó que poseen un nivel de desarrollo incipiente en las competencias en sostenibilidad y manejo adecuado de RAEE. En consecuencia, se realizaron diversas acciones en pro de aportar al cumplimiento de metas sostenibles y complementar su formación integral incluyendo al contenido de la asignatura aspectos de sostenibilidad y manejo adecuado de RAEE.

Palabras clave: educación; sostenibilidad; RAEE; tecnología e informática; contaminación.

\begin{abstract}
The purpose of this article is to reflect on the level of knowledge of sustainability the students of Licenciature of Informatic of Universidad del Magdalena have, and their responsibility in promoting proper management of WEEE. The fact that technological advances are only regarded because of their benefits and innovation is concerning. The consequences of these developments are not often considered, such as the waste they generate, which is made up of both valuable and potentially dangerous materials. Among the main theoretical references considered in this text are the SDG of UNESCO, the technical guidelines for proper management of WEEE, the Green Book of Colciencias, which promote the development

\footnotetext{
${ }^{1}$ Lic. Universidad del Magdalena, Santa Marta, Colombia. Correo electrónico: silviandreatorres@gmail.com ORCID: 0000-0002-8401-3305

${ }^{2}$ Lic. Universidad del Magdalena, Santa Marta, Colombia. Correo electrónico: salcedojulieth2711@gmail.com ORCID: 0000-0001-7216-8767

${ }^{3}$ Dra. en Ciencias de la Educación. Universidad del Magdalena, Santa Marta, Colombia. Correo electrónico: lbustamante@unimagdalena.edu. co ORCID: 0000-0002-4418-7845
} 
of competencies in sustainability as a cross-cutting issue in all educational levels. The reflections presented here arise from a descriptive research whose population were students of the course Fundamentals of Programming during 2018, They were the subject of a pilot test in which it was determined that they are in a stage of incipient development regarding sustainability competences and proper management of WEEE. Various actions were taken in order to achieve sustainable goals and complement their comprehensive training, by including contents related to key aspects of sustainability and proper handling of WEEE.

Keywords: Education; Sustainability; WEEE; Technology and Informatic; Contamination.

\section{INTRODUCCIÓN}

as tecnologías de la información y la comu-nicación (TIC) han traído consigo innumerables beneficios y con el pasar del tiempo han ayudado a mejorar y a facilitar la vida de las personas. Sin embargo, un factor poco considerado es la contaminación que causan los residuos tecnológicos cuando terminan su ciclo de vida útil. Los aparatos eléctricos y electrónicos (AEE) son aquellos que necesitan corriente eléctrica para poder funcionar, y pasan a ser residuos de aparatos eléctricos y electrónicos (RAEE) cuando han terminado su ciclo de vida útil o cuando su poseedor los considera obsoletos. Estos RAEE representan un peligro para el medio ambiente pues están compuestos por cientos de materiales tanto valiosos como potencialmente peligrosos, "que pueden poner en peligro la salud de las personas y el medio ambiente si no se manejan de manera adecuada" (Ministerio de Ambiente, Vivienda y Desarrollo Territorial de la República de Colombia, 2010, p.14).

Dada esta problemática medioambiental, distintas organizaciones como la United Nations Educational, Scientific and Cultural Organization (UNESCO) han hecho un llamado de alerta que consiste en integrar la educación para el desarrollo sostenible (EDS) a los distintos niveles educativos. Esto tiene como fin dar cumplimiento a los objetivos y metas de desarrollo sostenible para que en el 2030 "todos los alumnos adquieran los conocimientos teóricos y prácticos necesarios para promover el desarrollo sostenible, entre otras cosas mediante la educación para el desarrollo sostenible y la adopción de estilos de vida sostenibles" (Brown, 2017, p.1).

La EDS va de la mano con la educación de calidad integral y transformativa, pues esta "habilita a los educandos para tomar decisiones fundamentadas y adoptar medidas responsables en favor de la integridad del medio ambiente y la viabilidad de la economía" (UNESCO, 2018, p.1). Por ende, todos los tipos de instituciones educativas "pueden y deberían considerar su responsabilidad de abordar intensivamente temas de desarrollo sostenible, y de promover el desarrollo de las competencias de sostenibilidad" (Tang, 2017, p.7).

En este sentido, Colombia ha dado un paso para la renovación de la política nacional de ciencia, tecnología innovación $(\mathrm{CTeI})$ pues esta se viene desarrollando bajo un enfoque transformativo "cuyo propósito central es contribuir en la solución de los grandes desafíos sociales, económicos y ambientales que enfrenta nuestro país" (Vélez et al., 2018, p.13) mediante la transición de los actuales sistemas sociales y tecnológicos hacia unos más sostenibles.

Considerando que la búsqueda de soluciones a los grandes problemas sociales, económicos y ambientales del país exceden el alcance 
sectorial y constituyen por igual un desafío para los gobiernos nacional, regional y local, así como para el sector productivo, la academia y la sociedad civil, la adopción de un enfoque transformativo constituye un camino posible para el trabajo interinstitucional, multinivel y multiactor, para avanzar hacia la transformación (Vélez et al., 2018, p.13).

A partir de esta premisa, la educación empieza a ser un factor importante porque, como ente de transformación social, es el medio idóneo para empezar a desarrollar competencias sostenibles y conseguir el logro de los Objetivos de Desarrollo Sostenible (ODS), los cuales "conllevan un espíritu de colaboración y pragmatismo para elegir las mejores opciones con el fin de mejorar la vida de manera sostenible para las generaciones futuras" (PNUD, 2017, p.1). Esto solo es posible a través de la educación superior, pues según Minguet, Ull, Piñero y Martínez-Agut (2014):

La universidad debe formar a profesionales capaces de utilizar sus conocimientos, no solo en un contexto científico, sino también para dar respuesta a los problemas ambientales y a las necesidades sociales, tomando decisiones y realizando acciones coherentes con los valores de la sostenibilidad (p.138).

Debido al compromiso que poseen las instituciones de educación superior en cuanto a formación en sostenibilidad, es válido resaltar la importancia que esta tiene frente a la formación de los educadores, pues los futuros docentes deberían poseer las competencias profesionales que les permitan en su quehacer pedagógico realizar acciones sostenibles. Por lo tanto, como lo menciona Murga-Menoyo (2015), se "requiere que el profesorado de todos los niveles educativos, tanto en el sistema escolar como en la formación profesional y la universitaria, se comprometa activamente a imbuir en su práctica docente los principios y valores del desarrollo sostenible" (p.57). El objetivo de esto es implementar una pedagogía que transforme el actuar y el pensar de los individuos, contribuyendo al ejercicio de la EDS como instrumento indispensable para alcanzar los ODS planteados por la UNESCO.

Así pues, se debe concientizar en cuanto a que el compromiso no es solo de los futuros docentes; este va desde lo personal hasta la responsabilidad y la ética profesional, pues el llamado de adoptar acciones sostenibles está dirigido no solo a la docencia, sino a todas las carreras y a las distintas profesiones, con el fin de adoptar acciones ciudadanas responsables que no sean perjudiciales con el medio ambiente. En concordancia, Nava, Seijo y Mier (2014) hablan sobre el rol fundamental de la ética en relación con las actuaciones humanas debido a que es importante saber cómo el individuo en sociedad mantiene una actuación congruente en la vida ciudadana y en la vida profesional en pro del cuidado del medio ambiente.

Teniendo en cuenta que las universidades son las encargadas de formar y capacitar a los profesionales que necesita la sociedad, Sáenz, Plata, Holguín, Mora y Blanco (2017) señalan que las instituciones de educación superior están en la responsabilidad de abordar el tema de la sostenibilidad desde ámbitos como la docencia, la investigación, la extensión y la gestión, pues estos son los espacios en los que las universidades colombianas deberían adquirir compromisos ambientales.

Del mismo modo, Blanco-Portela y Benayas (2017) afirman que las universidades deberían generar cambios que involucren la transformación de sus funciones en docencia, investigación y extensión, en función de impulsar el cambio en las nuevas generaciones de profesionales 
que forman. Estos autores resaltan que la formación para la sostenibilidad requiere de dos grandes componentes institucionales: por un lado, los relacionados con el proceso formativo de todos los actores de la comunidad académica $y$, por otro, el respaldo institucional, expresado en una cultura organizacional comprometida e involucrada directamente en el cambio para la integración.

Ahora bien, teniendo claro que el tema de la sostenibilidad debe ser abarcado desde las distintas profesiones, se recomienda a las universidades acogerse a lo que propone la UNESCO: adoptar medidas y acciones sostenibles e involucrarse con la educación ambiental y la educación para el desarrollo sostenible, pues estas instituciones deben formar excelentes profesionales, es decir,

Personas competentes en la problemática ambiental, capaces de transmitir su conocimiento, plantear proyectos, y multiplicar procesos de educación ambiental a nivel local, regional y nacional en los diferentes sectores, con el fin de que el ciudadano común adopte hábitos diarios para el cuidado del ambiente y la preservación de los recursos naturales (PitaMorales, 2016, p.123).

Por otro lado, abordando la EDS, es necesario comentar que es un factor clave para el logro de los distintos ODS. Por ende, el tema del consumo sostenible es un elemento trascendente que vale la pena nombrar, pues actualmente la humanidad ha desarrollado una cultura basada en los excesos: para este caso, los relacionados con los aparatos tecnológicos debido a la constante innovación TIC de los últimos tiempos. Es por esta razón que a través de la EDS se pretende generar conciencia, actuaciones sociales y ambientales responsables a fin de mejorar la calidad de vida, no solo de las personas en la actualidad, sino de las generaciones venideras.
Otro aspecto importante con respecto a la EDS es lo mencionado por Vilches y Gil (2012): "Reorientar los programas de elaboración de planes de estudio y formación de docentes, con miras a integrar la EDS en los programas de formación inicial y de perfeccionamiento para docentes en funciones" (p.40). En ese orden de ideas, a partir de los programas de formación de cada institución educativa se deben ir transformando poco a poco las bases académicas en aras de ir incorporando la sostenibilidad al ámbito académico, realizando ajustes en el currículo del sistema actual.

A partir de estas apreciaciones y teniendo en cuenta que es deseable que los futuros licenciados posean competencias en sostenibilidad, se optó por realizar una investigación que tuviera como objetivo determinar el nivel de conocimiento sobre sostenibilidad y manejo adecuado de los RAEE que poseen los estudiantes de la Licenciatura en Informática debido a que se considera que son los llamados a concientizar sobre esta problemática desde la educación básica, como parte de la formación en el área de tecnología e informática.

\section{ACCIONES PARA CONTRIBUIR A LA FORMACIÓN EN SOSTENIBILIDAD}

El cuarto ODS, "Educación de Calidad", propone como meta al 2030:

Asegurar que todos los alumnos adquieran los conocimientos teóricos y prácticos necesarios para promover el desarrollo sostenible, entre otras cosas mediante la educación para el desarrollo sostenible y los estilos de vida sostenibles, los derechos humanos, la igualdad de género, la promoción de una cultura de paz y no violencia, la ciudadanía mundial y la valoración de la diversidad cultural y la contribución 
de la cultura al desarrollo sostenible (Brown, 2017, p.1).

Teniendo esto en cuenta, desde el programa de Licenciatura en Informática se han realizado una serie de acciones en pro de favorecer el cumplimiento de la meta planteada. Una primera acción fue la realización de una prueba piloto que buscaba establecer y valorar los conocimientos sobre sostenibilidad y manejo adecuado de RAEE. Este instrumento fue aplicado a treinta y seis estudiantes de Licenciatura en Informática de quinto semestre que cursaban la asignatura Fundamentos de Programación en el primer período del 2018. El instrumento constaba de siete interrogantes que iban desde la concepción de sostenibilidad hasta la identificación de los RAEE, así como sus criterios de clasificación.

Del mismo modo, en el marco de la asignatura Fundamentos de Programación, el docente y los estudiantes realizaron el curso virtual "Escuela de sostenibilidad" de Bancolombia, que se puede encontrar en el siguiente enlace: https:// www.escuelasostenibilidad.com/es. La capacitación consiste en realizar un recorrido de nueve lecciones por la comunidad del Islote de Santa Cruz, cada una de las cuales está representada por un integrante de la población considerado "maestro en sostenibilidad". Así pues, el fin del curso es aprender de esta comunidad a realizar acciones cotidianas sostenibles como reutilizar el agua, recolectar adecuadamente las basuras, ahorrar energía y formar en actitudes y valores sostenibles para finalmente poder "graduarse" de la escuela de sostenibilidad.

En la primera lección, denominada "La pesca que alcanza para uno alcanza para todos", lleva a reflexionar sobre la importancia que tiene para las comunidades compartir en pro del beneficio de los demás sin dejar de lado el cuidado del medio ambiente. En este caso, el "maestro en compartir" busca no solo el bienestar de aquel que no posee recursos o alimentos, sino el del medio ambiente, pues el aumento de la población de pescadores y de la demanda del producto del mar perjudica el principal sustento de la comunidad.

Por otro lado, la segunda lección, "Hacer la vaca para pagar la luz", brinda una enseñanza fundamental en cuanto a la colaboración que se debe destacar en las comunidades a la hora de buscar soluciones a las distintas necesidades. La tercera lección, "Educar el futuro de la isla", nos lleva a afianzar una vez más el valor de la educación como medio de transformación social. En este caso, las representantes de la lección son denominadas "maestras de la convivencia" dado que promueven en los niños de la isla una cultura de no violencia enriquecida por modales y valores. Esta lección permite conocer la importancia de educar a la niñez y de entender que ellos son el futuro de las sociedades.

En la cuarta lección, "Un trabajo me ayuda a mí, el otro ayuda a la isla", el "maestro en ayudar a la comunidad" nos invita a tomar conciencia, a cambiar nuestra conducta en cuanto a las acciones que realizamos en beneficio del medio ambiente, y a tomar iniciativas de cuidado y protección del medio que nos rodea con el fin de aportar a la sociedad en la que vivimos. La quinta lección, denominada "Tanta agua, tan pocos peces", conlleva a reflexionar sobre las acciones que están afectando la fauna y el ecosistema marino pues el crecimiento de la comunidad pescadora ha ocasionado prácticas de pesca no sustentables que de no ser corregidas perjudicarían cada vez más los recursos que brinda el mar.

La sexta lección, denominada "El agua es oro", nos lleva a pensar sobre el valor y cuidado de los recursos hídricos; nos motiva a valorar y a 
darle un mejor uso al agua y a aprovechar cada uno de sus beneficios, pero, sobre todo, a actuar de manera sostenible. La séptima lección, titulada "El voluntario que entrena a las promesas de la isla", brinda la oportunidad de reflexionar acerca de lo que estamos aportando a nuestra sociedad, motiva a creer en las capacidades de los niños y, especialmente, propone un ejemplo que motiva a ser voluntarios, constructores de sociedad, transformadores de estilos de vida y de las comunidades.

En la octava lección, "En esta tienda no se desprecia ni un poquito", la "maestra en consumo responsable" nos incentiva a reflexionar acerca de la importante labor que realizan los pequeños comerciantes a la hora de ajustar sus servicios para suplir las necesidades de las personas que poseen menos recursos. Por último, la novena lección, "Qué bueno es empezar con una gran bienvenida al mundo", destaca cómo, a pesar de que podría pensarse que nacer en la isla es una desventaja por las condiciones de precariedad, el hecho de que los partos sean naturales les permite a los niños recibir lactancia materna exclusiva desde sus primeras horas de vida, algo que es difícil de lograr en las ciudades, donde los niños son separados de sus madres y en algunas ocasiones alimentados con fórmulas lácteas. Este fenómeno urbano es contrario a lo que sugiere la Organización Mundial de la Salud (OMS) en cuanto a garantizar que el niño reciba lactancia materna durante sus primeras horas de vida para garantizar que reciba el calostro, el cual lo inmuniza y alimenta de forma segura y sustentable.

Dentro de este curso, y teniendo en cuenta lo mencionado por Minguet y Solís (2009) "Todos los estudiantes universitarios tendrían que formarse en sus campos de especialización de acuerdo con criterios y valores relacionados con la sostenibilidad" (p.5)—, se optó por aportar a la construcción de historias animadas sobre las diferentes profesiones que ofrece la Universidad del Magdalena agregándole un aspecto relevante: lo que cada profesión le puede aportar al desarrollo sostenible. En la tabla 1 se evidencia la contribución de cada programa.

La información de la tabla 1 permite reconocer cómo desde cada profesión se pueden realizar aportes a la sostenibilidad. Esto puede sensibilizar a los estudiantes para que en su próximo quehacer profesional incorporen los lineamientos estipulados en los ODS con miras a un futuro sustentable, es decir, que se reconozcan como los próximos tomadores de decisiones y quienes tienen la responsabilidad de concientizar a sus familias y colegas con las acciones de su labor diaria sobre la importancia de un futuro donde todos contribuyan a preservar el medio ambiente. Estas acciones pueden traer una mejor calidad de vida en la medida en que todas las personas contribuyan, y una de las maneras de lograrlo es desde las aulas de clase, comenzando a vivenciar la sostenibilidad de modo transversal en todas las asignaturas. 
Tabla 1. Contribución de cada profesión a la sostenibilidad.

\begin{tabular}{|l|l|}
\hline \multicolumn{1}{|c|}{ Programa } & \multicolumn{1}{c|}{ Contribución a la sostenibilidad } \\
\hline $\begin{array}{l}\text { Licenciatura en } \\
\text { Educación Infantil }\end{array}$ & $\begin{array}{l}\text { Estilos de vida saludables, hábitos de higiene, alimentación saludable, cuidado } \\
\text { personal, entre otros. }\end{array}$ \\
\hline $\begin{array}{l}\text { Licenciatura en } \\
\text { Informática }\end{array}$ & $\begin{array}{l}\text { Evitar impresiones innecesarias, usar ambas caras del papel, manejo adecuado } \\
\text { de RAEE. }\end{array}$ \\
\hline Medicina y Enfermería & $\begin{array}{l}\text { Hábitos de alimentación sanos, mayor actividad física, estilos de vida menos } \\
\text { sedentarios, disminución de contaminación, entre otros. }\end{array}$ \\
\hline Odontología & $\begin{array}{l}\text { Buenos hábitos de salud bucodental, responsabilidad en la recolección y } \\
\text { tratamiento de los residuos odontológicos. }\end{array}$ \\
\hline Psicología & Bienestar psicosocial que genera participar como voluntario para ayudar a otros. \\
\hline Biología & Campañas informativas de recolección y sensibilización. \\
\hline $\begin{array}{l}\text { Administración de } \\
\text { Empresas }\end{array}$ & Creación de empresas sostenibles en lo económico, lo ambiental y social. \\
\hline $\begin{array}{l}\text { Negocios } \\
\text { Internacionales }\end{array}$ & Generación de empleos y mejores oportunidades para la sociedad. \\
\hline Contaduría & Satisfacer las necesidades actuales sin poner en peligro los recursos del futuro. \\
\hline Ingeniería de Sistemas & Desarrollo de softwares especializados, promoción de sistemas online. \\
\hline Ingeniería Ambiental & Construcción de proyectos responsables con el entorno. \\
\hline Antropología & Asumir modos de vida que involucren creencias y tradiciones sostenibles. \\
\hline Cine y Audiovisuales & Producción audiovisual y cinematográfica respetuosa con el medio ambiente. \\
\hline
\end{tabular}

Fuente: elaboración propia.

En este espacio también se presentó una aplicación para conocer la cultura arhuaca en la que se socializa información relevante sobre el equilibrio que se debe mantener para respetar y preservar el medio ambiente y la importancia de las generaciones ancestrales. Así mismo se da a conocer el respeto que esta cultura muestra por la madre tierra, además de destacarla como un ejemplo de solidaridad ambiental.

Un último punto con el que se aportó al cumplimiento del tema de sostenibilidad fue la creación de aplicaciones educativas con la herramienta Scratch 2.0. Los estudiantes de la cátedra de Fundamentos de Programación realizaron sus trabajos con base en información relacionada con los RAEE, de manera que estas aplicaciones abordaban temas como el impacto ambiental que tienen estos residuos, la utilidad del reúso, las recomendaciones y lineamientos para la recolección de RAEE, los componentes químicos peligrosos que se encuentran presentes en estos residuos, la reutilización y el reciclaje de pantallas de cristal, residuos de computadores, acumuladores de pilas, periféricos de entrada y salida, juegos eléctricos y electrónicos, entre otros. Todo esto es de vital importancia puesto que, como futuros licenciados, los estudiantes deben tener claras las competencias sobre sostenibilidad y el adecuado manejo del residuo de aparatos eléctricos y electrónicos, es decir, tener claro que manejar adecuadamente este tipo de residuos aporta de manera positiva a un mundo sostenible. 
En este sentido, los docentes en formación que hacen parte de esta asignatura pretenden dar a conocer la problemática ambiental causada por la contaminación de RAEE dado que, como lo menciona el Ministerio de Ambiente, Vivienda y Desarrollo Territorial (2010) en el documento sobre los lineamientos técnicos para el manejo de residuos de aparatos eléctricos y electrónicos, "para el 2007 se estimó una generación entre 6 000 y 9000 toneladas de estos residuos y para el 2013 se esperaba entre 80000 y 140000 toneladas" (p.7). Este panorama es relevante para los docentes en formación pues los principales elementos y aparatos TIC de los que hacen uso en su quehacer pedagógico contienen grandes cantidades de componentes contaminantes $y$, por ende, desde su formación académica deben comenzar a adquirir competencias que les permitan hacer un buen manejo de RAEE pero, sobre todo, adquirir y desarrollar hábitos sostenibles.

\section{REFLEXIONES A PARTIR DE LA ACCIONES PARA CONTRIBUIR A LA FORMACIÓN EN SOSTENIBILIDAD}

A partir de las diferentes intervenciones realizadas, es necesario mencionar que la prueba piloto evidencia que los estudiantes del programa de Licenciatura en Informática de la Universidad del Magdalena poseen un nivel de desarrollo incipiente en las competencias en sostenibilidad y manejo adecuado de RAEE. Las respuestas a preguntas como “ ¿qué entiende usted por sostenibilidad?”, “ ¿cree usted que los residuos de aparatos eléctricos y electrónicos son contaminantes?” o “ ¿considera necesaria la recolección de aparatos electrónicos después de su ciclo de uso?" dan pie para realizar un análisis pues la mayoría de los estudiantes no identifican el término "sostenibilidad" ni todo lo que este atañe. Asimismo, las siglas RAEE son desconocidas para gran parte de ellos.
No obstante, algunos de los estudiantes consideran que los RAEE son contaminantes, pero difieren a la hora de contestar si creen necesaria la recolección de estos después de su ciclo de vida. De hecho, un gran porcentaje de los participantes no considera que estos residuos deberían ser recolectados de manera diferente al resto de los residuos, lo que denota que existen vacíos considerables al respecto. A continuación, se presentan algunas gráficas con los resultados encontrados después de la indagación.

Teniendo en cuenta que Tang (2017) enfatiza en que "Todos los alumnos adquieran los conocimientos teóricos y prácticos necesarios para promover el desarrollo sostenible mediante la educación para el desarrollo, los estilos de vida y la contribución a una cultura que promueva el desarrollo sostenible" (p.7), los datos de la figura 1 generan preocupación debido a que el $75 \%$ de los estudiantes de la Licenciatura en Informática no conocen el término "sostenibilidad". Siguiendo entonces la premisa presentada por la UNESCO es necesario reflexionar a partir de la práctica pedagógica pues, al desconocer el término, es posible que los participantes no reconozcan dentro de su labor la importancia de la sostenibilidad.

Figura 1. Concepción de sostenibilidad.

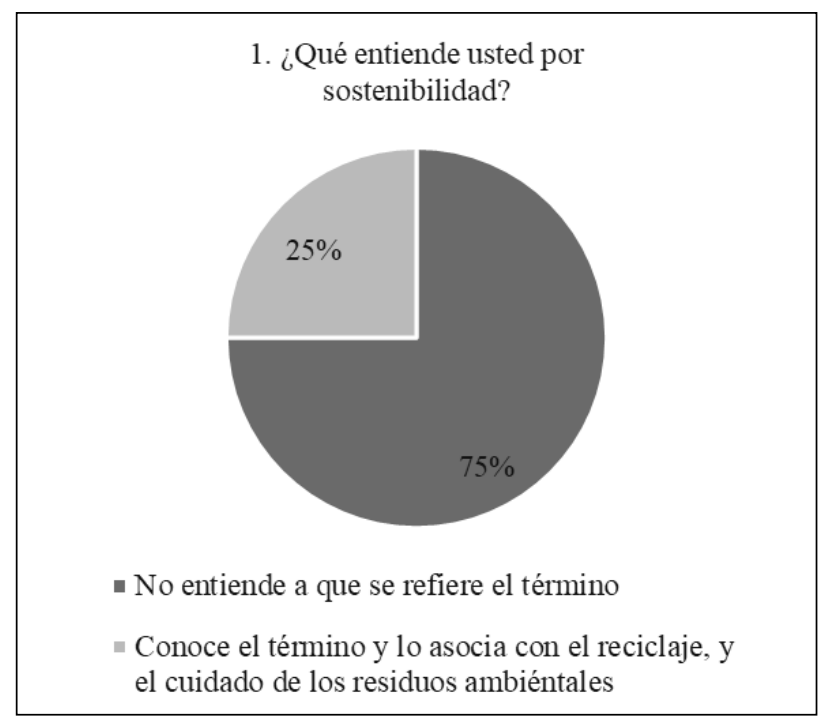

Fuente: elaboración propia. 
Por otro lado, es necesario mencionar que la sostenibilidad relaciona tres aspectos fundamentales: el aspecto económico, el social y el ambiental. Estos deben mantener un equilibrio pues están asociados de tal manera que uno depende del otro, es decir, las prácticas deben ser económicamente rentables, sin olvidar iniciativas sociales y ambientalmente responsables. En este sentido, si observamos que la mayoría de los participantes no entiende a qué se refiere el término y el 25 $\%$ lo asocia solo con el cuidado de los recursos ambientales, podría decirse que es posible que los futuros licenciados en informática no posean las concepciones necesarias para promover actuaciones sostenibles dentro de las aulas de clase.

De igual forma, si visualizamos este punto desde la perspectiva de formación profesional, podría decirse que estos porcentajes representan de una $\mathrm{u}$ otra manera falencias dentro de la formación profesional de los futuros licenciados en informática pues, como se ha mencionado anteriormente, las instituciones educativas, en este caso las universidades, deben promover a través de la EDS actuaciones que permitan gestores de sostenibilidad.

Figura 2. Contaminación de RAEE.

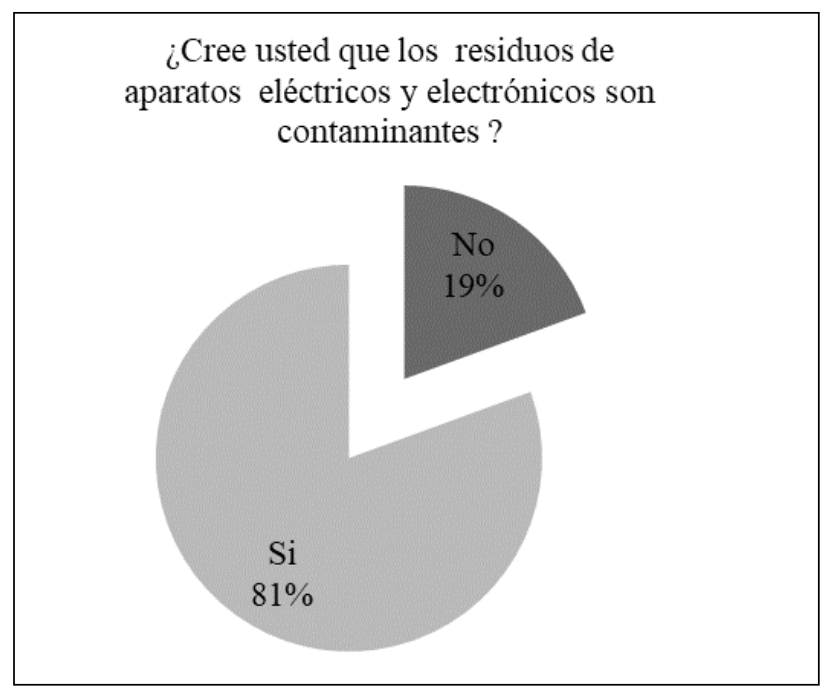

Fuente: elaboración propia.
La figura 2 nos presenta un aspecto importante: la contaminación por RAEE. En este caso podemos denotar que el $81 \%$ de los participantes los considera contaminantes, siendo esto relevante pues da a conocer que son conscientes de una $\mathrm{u}$ otra manera de que los residuos tecnológicos pueden causar daños en la salud de los seres humanos y afectar de manera irremediable el medio ambiente. Del mismo modo, estos resultados nos llevan a contemplar que un pequeño porcentaje de los estudiantes que realizaron la prueba no consideran que este tipo de residuos sean contaminantes, lo que causa preocupación pues quiere decir que es posible que no tengan un consumo y desecho responsable de los diferentes artefactos tecnológicos de su vida cotidiana.

Figura 3. Recolección de AEE después de su uso.

¿Considera necesaria la recolección de aparatos electrónicos después de su ciclo de uso?

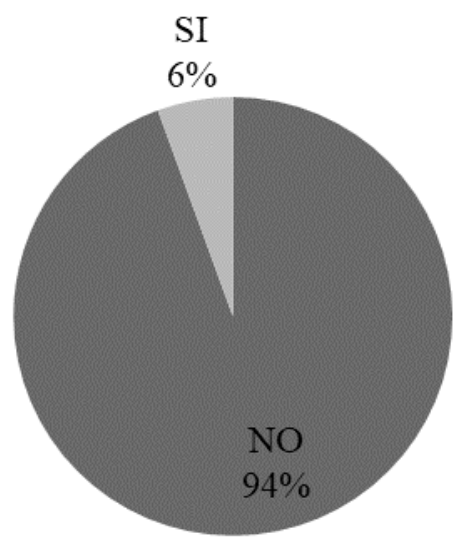

Fuente: elaboración propia.

Finalmente, los resultados de la figura 3 generan un contraste en relación con los resultados de la figura 2. Así como se mencionó que el $81 \%$ los estudiantes que participaron de esta prueba piloto consideraban los RAEE como contaminantes, en este apartado, que tiene que ver con la recolección de los AEE después de su ciclo de 
uso, un $94 \%$ afirma no considerarla necesaria, lo que nos lleva a reflexionar sobre la discordancia de estos porcentajes: si la mayoría de los estudiantes consideran que los RAEE son contaminantes, ¿por qué no será necesaria su recolección? Al no encontrarles una explicación coherente a estos resultados, podría decirse que es posible que los participantes no sean conscientes de que somos una especie que genera desequilibrio en el medio ambiente dadas las malas prácticas que se realizan en la cotidianidad pues, como se ha afirmado anteriormente, los RAEE poseen algunos componentes considerados potencialmente peligrosos que ocasionan daño al medio ambiente, mucho mayor incluso que el producido por el plástico.

Teniendo en cuenta lo anterior, las actuaciones sostenibles deben ser promovidas sin olvidar que cada individuo debe poseer unas concepciones que le permitan desarrollar actitudes y aptitudes en pro de la conservación del medio ambiente y el bienestar de la sociedad.

\section{CONCLUSIONES}

A lo largo del desarrollo de este escrito se evidencia la importancia de tener en cuenta los tres aspectos que hacen posible tener actuaciones sostenibles: lo económico, lo ambiental y lo social. En diversas ocasiones se comete el error de asociar la sostenibilidad solo con el manejo económico y se excluyen los aspectos ambientales y sociales. Esto ocasiona que se les reste importancia a los altos niveles de contaminación medioambiental y a la responsabilidad que poseen las sociedades.

Para fomentar la responsabilidad que poseen las sociedades, surge la EDS como parte del cuarto ODS. En atención a ello, todos los docentes, en los diferentes niveles educativos, deberían contribuir a generar conciencia en cuanto al compromiso que todos tenemos por preservar el medio ambiente y contribuir al cumplimiento de las metas al 2030.

En particular, la educación universitaria se encuentra rezagada en el cumplimiento de estas metas, lo que genera gran preocupación al entender que son los estudiantes universitarios los siguientes profesionales y, por ende, quienes en el corto o mediano plazo serán quienes tomarán las decisiones. Por esta razón urge su formación en sostenibilidad con miras a desacelerar la contaminación ambiental y generar las condiciones para propiciar la restauración del medio ambiente.

Si bien desde las diferentes profesiones se puede contribuir al desarrollo sostenible, tal como lo develaron los estudiantes a través de las animaciones que crearon para las diferentes carreras que ofrece la universidad, se considera trascendental la labor del licenciado en Informática, en su rol como docente del área de tecnología e informática, la cual es considerada un área transversal al servicio del resto de las disciplinas.

Siendo el área de tecnología e informática transversal, tal como lo es la sostenibilidad, aunado al poco conocimiento en cuanto a la gestión adecuada de RAEE, se considera que empoderar a los egresados del programa de la Licenciatura en Informática de competencias en sostenibilidad podría contribuir en gran manera con el desarrollo sostenible.

\section{REFERENCIAS BIBLIOGRÁFICAS}

Blanco-Portela, N. y Benayas, J. (2017). Formación para la sostenibilidad en las universidades latinoamericanas: análisis de resultados del proyecto RISU. Enseñanza de Las Ciencias: Revista de Investigación y Experiencias Didácticas, Extra, 3155-3162. Recuperado de https:// www.raco.cat/index.php/Ensenanza/article/ view/337036/427906 
Brown, M. (2017). Educación para el desarrollo sostenible. Quito, Ecuador: Organización de las Naciones Unidas para la Educación, la Ciencia y la Cultura. Recuperado de http:// www.unesco.org/new/es/quito/education/ educacion-para-el-desarrollo-sostenible/

Ministerio de Ambiente, Vivienda y Desarrollo Territorial de la República de Colombia. (2010). Lineamientos técnicos para el manejo de residuos de aparatos eléctricos y electrónicos. Recuperado de http://www.residuoselectronicos.net/wp-content/ uploads/2012/03/Guia_RAEE_MADS_2011reducida.pdf

Minguet, P., Ull, M., Piñero, A. y Martínez-Agut, M. (2014). La sostenibilidad en la formación universitaria: desafíos y oportunidades. Educación XX1, 17(1), 133-158. doi: 10.5944/ educxx1.17.1.10708

Minguet, P. A. y Solís, A. U. (2009). La formación de competencias básicas para el desarrollo sostenible: el papel de la Universidad. Revista de Educación, (1), 219-237.

Murga-Menoyo, M. Á. (2015). Competencias para el desarrollo sostenible: las capacidades, actitudes y valores meta de la educación en el marco de la Agenda global post-2015. Foro de Educación, 13(19), 55-83. doi:10.14516/fde.2015.013.019.004

Nava, D., Seijo, C. y Mier, C. (2014). Inteligencias múltiples: característica congruente del liderazgo ético en entidades financieras. Praxis, 10, 91-99. doi:10.21676/23897856.1361.

Pita-Morales, L. A. (2016). Línea de tiempo: educación ambiental en Colombia. Praxis, 12, 118-125. doi:10.21676/23897856.1853
PNUD. (2017). Objetivos de Desarrollo Sostenible. Nueva York, USA: Programa de las Naciones Unidas para el Desarrollo. Recuperado de: https://www.undp.org/content/undp/es/home/ sustainable-development-goals.html

SáenzZapata, O., Plata Rangel, Á. M., Holguín Aguirre, M. T., Mora Penagos, W. M. y Blanco Portela, N. (2017). Institucionalización del compromiso ambiental de las universidades colombianas. Civilizar Ciencias Sociales y Humanas, 17, 189208. doi:10.22518/16578953.908

Tang, Q. (2017). Educación para los Objetivos de Desarrollo Sostenible: objetivos de aprendizaje; 2017. Recuperado de https://unesdoc.unesco.org/ ark:/48223/pf0000252423/PDF/252423spa.pdf. multi

UNESCO. (2018). ¿Qué es la Educación para el Desarrollo Sostenible?. Nueva York, USA: Organización de las Naciones Unidas para la Educación, la Ciencia y la Cultural. Recuperado de https://es.unesco.org/ themes/educacion-desarrollo-sostenible/ comprender-EDS

Vélez, M., Chavarro, A., Hernandez, A., Niño, A., Tovar, G. y Montenegro, I. (2018). Libro Verde 2030 Política de ciencia e innovación para el Desarrollo Sostenible en Colombia. Recuperado de http://www.apropiate.colciencias.gov.co/sites/ default/files/2018-08/libro_verde.pdf

Vilches, A. y Gil Pérez, D. (2012). La educación para la sostenibilidad en la universidad: el reto de la formación del profesorado. Profesorado, Revista de Currículum y Formación de Profesorado, 16(2), 25-43. 